\title{
Central motor conduction time in malnourished children
}

\author{
S K Tamer, S Misra, S Jaiswal
}

\begin{abstract}
The functional status of the descending motor pathways was assessed in malnourished children using transcranial electromagnetic stimulation of the cortex. Twenty children with different severities of malnutrition and 20 control subjects were studied electrophysiologically. The circular coil of a Dentac MAG 2 magnetic stimulator was applied tangentially over the vertex to stimulate the cortex. The muscle evoked potential in the children's arms was recorded from the abductor pollicis brevis muscle and in their legs from the extensor digitorum brevis muscle on both sides of the body using surface electrodes. The muscle evoked potential of the abductor pollicis brevis and extensor digitorum brevis muscles was further obtained using root stimulation by applying the coil at the cervical and lumbar spine, respectively. The indices of cortical threshold, cortical latency, and central motor conduction time (ms) were evaluated in both arm and leg muscles on both sides. The results showed an increased cortical threshold (mean (SD) 1232.5 $(134.06)$ in the study group $v 1147.5(99.31)$ in the control group) for the abductor pollicis brevis muscle and for the extensor digitorum brevis muscle (1325.00 (115.70) in the study group $v 1190.0(125.24)$ in the control group). Similarly, significant prolongation of the central motor conduction time (ms) (study group $6.67(0.91) v$ control group $5.71(0.74)$ ) in the abductor pollicis brevis muscle was seen in malnourished children.

(Arch Dis Child 1997;77:323-325)
\end{abstract}

Division of Neurology, Institute of Medical

Sciences BHU,

Varanasi, and Section

of Paediatric

Neurology,

Department of

Neurosciences, JLN

Hospital and Research

Centre, Bhilai MP

490006, India

$S$ K Tamer

S Misra

S Jaiswal

Correspondence to:

Dr S K Tamer, Department

of Pediatrics and Neurology,

644, 'Samidha', Sindhia

Nagar, PO-Durg MP

491001, India.

Accepted 17 February 1997 superficial layers of the cortex. By stimulating the motor cortex, the muscle evoked potential is obtained, which can be measured by surface electromyograph electrodes. This study assessed the functional aspects of the central motor pathway in malnourished children compared with children who were not malnourished.

\section{Subjects and methods}

The subjects in this study were 40 children aged 1-5 years. Of these, 20 children who were malnourished served as the study group, whereas the other 20 , who were within the normal range in weight for their age, were used as controls. Body weight was used to grade the severity of malnutrition as recommended by the Nutrition Sub-Committee of the Indian Academy of Pediatrics. ${ }^{4}$ Each child was weighed with minimum clothing on a spring balance which measured to the nearest $0.5 \mathrm{~kg}$. The weight of the 50th Harvard centile was taken as a standard and each child's weight was compared with the standard expressed as a percentage and graded as follows: $>80 \%$ of standard, control; $61-80 \%$ of standard, malnourished grade 1, 12 children; and $\leqslant 60 \%$ of standard, severely malnourished, grade 2 , eight children.

Each child enrolled for the study was evaluated according to a pretested proforma. Information collected included rural or urban residence, literacy, occupation of father, size of the family, and number of siblings. Immunisation status and feeding history were recorded for each child. The historical profile of these children included the presenting complaint for which the child had attended hospital. Minor ailments such as skin infection, acute respiratory infection, and viral febrile illness were recorded. Patients with serious systemic diseases and particularly those affecting the nervous system were excluded. Similarly, past significant history was recorded. Neurological illnesses such as seizures, inflammatory brain diseases, paresis, and other neurological deficits were specifically queried and children were excluded from the study in the presence of any such past or present illness. Information about their antenatal history and perinatal events including exposure to drugs, irradiation, maternal infection, mode of delivery, birth asphyxia, and resuscitation was recorded with a view to pinpointing other environmental insults to the fetuses and infants during these critical periods. Bedside clinical assessments of significant milestones of development were carried out for each child and a gross delay in various fields was taken as an exclusion criteria.

A thorough physical examination included vital parameters such as pulse, temperature 
Table 1 Age, weight, degree of malnutrition, and gender distribution of study group and controls

\begin{tabular}{lll}
\hline & $\begin{array}{l}\text { Malnourished children } \\
(n=20)\end{array}$ & $\begin{array}{l}\text { Control group } \\
(n=20)\end{array}$ \\
\hline Mean (SD) age (years) & $3.2(1.3)$ & $3.3(1.3)$ \\
Mean (SD) weight (kg) & $10.15(3.11)$ & $13.55(2.59)$ \\
Male:female ratio & $12: 8$ & $13: 7$ \\
Mean (SD) \% of standard weight & $63.3(10.4)$ & $85.1(4.0)$ \\
No 61-80\% standard weight (grade 1) & 12 & 0 \\
No $\leqslant 60 \%$ standard weight (grade 2) & 8 & 0 \\
\hline
\end{tabular}

and respiration, skull morphology, sutures, fontanelle, eyes, skin, extremities, skeletal system, and lymphadenopathy. Neurocutaneous markers and congenital anomalies were looked for. The systemic examination included particular emphasis on the nervous system, including mental function, cranial nerves (including fundus), the motor system, reflexes, cerebellar signs, and any other abnormalities.

All electrophysiological investigations were carried out in our laboratory at the Division of Neurology, Institute of Medical Sciences, BHU, Varanasi on a Neuromatic 2000 two channel digital electromyograph system attached to a Dentac MAG 2 magnetic stimulator. Younger children aged 1-2 years were allowed to go to natural sleep, preferably after being fed in their mother's lap. Hypnotic drugs and sedatives were avoided as it is known that almost all drugs that inhibit cerebral activity, such as anticonvulsant, hypnotic, and most anaesthetic drugs, reduce the amplitude of muscle evoked potentials and increase the cortical threshold. A circular magnetic coil was used to stimulate the cortex and it was applied tangentially over the vertex region to obtain the muscle evoked potential in the arm muscle (abductor pollicis brevis). To obtain the muscle evoked potential of the leg muscle (extensor digitorum brevis) the suggested point of stimulation $(7 \mathrm{~cm}$ anterior and $2-4 \mathrm{~cm}$ lateral to the $\mathrm{Cz}$ point) was not valid for our subjects and several attempts were needed to produce muscle evoked potentials by repositioning the coil on the vertex. Initially, the muscle was kept relaxed and a DISA 13k60 surface electrode was applied to obtain the muscle evoked potential. All the children needed a facilitation process, which consisted of voluntary contrac-

Table 2 Cortical threshold required to induce muscle evoked potential in abductor pollicis brevis muscle

\begin{tabular}{lll}
\hline & $\begin{array}{l}\text { Mean }(S D) \\
\text { cortical threshold }(T)\end{array}$ & $\begin{array}{l}\text { Mean (SD) } \\
\text { \% of maximum output }\end{array}$ \\
\hline Control group ( $\mathrm{n}=20)$ & $1147(99)$ & $76.3(6.4)$ \\
Malnourished group $(\mathrm{n}=20)$ & $1233(134)^{\star}$ & $82.0(9.0)^{\star}$ \\
Malnourished, grade $1(\mathrm{n}=12)$ & $1192(138)$ & $79.4(9.4)$ \\
Malnourished, grade 2 $(\mathrm{n}=8)$ & $1294(108)^{\star \star}$ & $85.9(7.2)^{\star \star}$ \\
\hline
\end{tabular}

${ }^{\star} \mathrm{p}<0.05 v$ control group; ${ }^{\star \star} \mathrm{p}<0.01 v$ malnourished grade 1 .

Table 3 Cortical threshold required to induce muscle evoked potential in extensor digitorum brevis muscle

\begin{tabular}{lll}
\hline & $\begin{array}{l}\text { Mean }(S D) \\
\text { cortical threshold }(T)\end{array}$ & $\begin{array}{l}\text { Mean }(S D) \\
\% \text { of maximum output }\end{array}$ \\
\hline Control group $(\mathrm{n}=20)$ & $1190(125)$ & $79.1(8.3)$ \\
Malnourished group $(\mathrm{n}=20)$ & $1325(116)^{\star}$ & $88.2(7.8)^{\star \star}$ \\
Malnourished, grade $1(\mathrm{n}=12)$ & $1279(112)^{\star \star \star}$ & $85.2(7.7)^{\star \star \star}$ \\
Malnourished, grade 2 $(\mathrm{n}=8)$ & $1394(82)^{\star \star \star \star}$ & $92.7(5.6)^{\star \star \star \star}$ \\
\hline${ }^{\star} \mathrm{p}<0.01 ;{ }^{\star \star} \mathrm{p}<0.001 ;{ }^{\star \star \star} \mathrm{p}<0.05 ;{ }^{\star \star \star \star} \mathrm{p}<0.001$, all $v$ respective control group.
\end{tabular}

${ }^{\star} \mathrm{p}<0.01 ;{ }^{\star \star} \mathrm{p}<0.001 ;^{\star \star \star} \mathrm{p}<0.05 ;^{\star \star \star \star} \mathrm{p}<0.001$, all $v$ respective control group. tion of the muscle to a moderate degree, or passive contraction in younger children, again to a moderate degree, to induce the muscle evoked potential. Root stimulation was used to obtain the muscle evoked potential by applying the coil at the cervical and lumbar spine for the arm and leg, respectively. This was easier in our subjects who required less stimulus intensity. The muscle evoked potential values obtained from cortical and root stimulation were analysed for latency (ms) and amplitude (mV) (measured from peak to peak positive and negative deflection). The latency from cortical stimulation to the onset of the muscle evoked potential was recorded as the cortical latency, whereas the latency from root stimulation to the onset of the muscle evoked potential was recorded as the root latency.

Several methods have been suggested to determine the central motor conduction time. $^{5-7}$ In this work it was taken as the difference between the cortical latency and the root latency. Muscle evoked potentials were recorded from the abductor pollicis brevis and extensor digitorum brevis muscles on both sides. The cortical threshold was defined as the percentage of maximum output of stimulus intensity (1500 tesla units) required to induce an evoked response of $>20 \mu \mathrm{V}$ in amplitude. The cortical latency and central motor conduction time indices were evaluated for the study and control groups.

As there was no statistical difference in the results between the two sides, the results from the left side were used throughout the study.

Statistical comparison by Student's $t$ test and the $\chi^{2}$ test, and the trend of parameters by the correlation coefficient test of significance, were used where applicable. We tested whether the severity of malnutrition had any impact on these indices.

\section{Results}

Table 1 gives the mean (SD) age, weights, degree of malnutrition, and gender distribution of malnourished children and controls. The socioepidemiological factors of these children were similar in the two groups.

A significantly higher threshold was required to induce the muscle evoked potential in the arms (table 2) and in the legs (table 3) in malnourished children. The more severe the malnutrition, the higher the threshold to induce the muscle evoked potential.

The overall cortical excitability was reduced significantly in malnourished children compared with the control group as measured by an increased cortical threshold for both muscles in arms and legs. A trend of decreasing cortical threshold with increasing age was observed. This was statistically significant in both study and control groups for both muscles studied ( $\mathrm{p}<0.001$ abductor pollicis brevis muscle, study group; $\mathrm{p}<0.01$ abductor pollicis brevis muscle; control group $\mathrm{p}<0.001$ extensor digitorum brevis muscle, study group; $p<0.01$ extensor digitorum brevis muscle, control group).

Malnourished children showed a prolonged cortical latency, central motor conduction 
Table 4 Comparison between study and control groups of various indices of abductor pollicis brevis muscle

\begin{tabular}{|c|c|c|c|}
\hline & $\begin{array}{l}\text { Mean (SD) } \\
\text { cortical latency (ms) }\end{array}$ & $\begin{array}{l}\text { Mean (SD) } \\
\text { amplitude }(m V)\end{array}$ & $\begin{array}{l}\text { Mean (SD) central } \\
\text { motor conduction } \\
\text { time (ms) }\end{array}$ \\
\hline Control group $(n=20)$ & $15(1)$ & $0.65(0.30)$ & $5.7(0.7)$ \\
\hline Malnourished group $(n=20)$ & $16(0.5)^{\star}$ & $0.43(0.23)^{\star \star}$ & $6.7(0.9)^{\star \star \star}$ \\
\hline
\end{tabular}

${ }^{\star} \mathrm{p}<0.0001 ;{ }^{\star \star} \mathrm{p}<0.02 ;{ }^{\star \star \star} \mathrm{p}<0.001 ;$ all $v$ control group.

time, and reduced amplitude of muscle evoked potential compared with the control group, and these differences were statistically significant in the arm muscles (table 4).

\section{Discussion}

Barker et al used magnetic stimulation of the motor cortex through the intact scalp to study the central motor pathways. ${ }^{6}$ This non-invasive technique allows the central motor function to be studied with precision and objectivity. The procedure has been safely used in children of different ages by Koh and Eyre. ${ }^{7}$

Stimulation of motor cortex generates an impulse which travels through the corticospinal tract to induce a muscle evoked potential in the target muscle. Cortical excitability as assessed by the cortical threshold and central motor conduction assessed by different indices of the muscle evoked potential are established ways of assessing the functional status of the corticospinal tract. ${ }^{5}$

We easily obtained muscle evoked potentials in all the children in this study; however, facilitation was required in all the children, as reported elsewhere, and this has been attributed to a lower cortical excitability than adults due to functional immaturity. ${ }^{7}$ The malnourished children had a significantly increased cortical threshold and prolonged cortical latencies and central motor conduction times; a prolonged latency to muscle evoked potential after electromagnetic stimulation of the cortex is a sensitive indicator of abnormality in the descending motor pathway. The cortical threshold, representing cortical excitability, might be related to the maturation process of the cortex. The relation with the maturation process at different ages has been studied in experimental primates, but data in humans are scant. ${ }^{378}$

Our findings also confirm higher cortical thresholds in infants and young children than in adults, as shown by Flament et al. ${ }^{9}$ Malnourished children also required a significantly higher cortical threshold to induce a muscle evoked potential, suggesting a dysfunction in the maturation process as a result of undernutrition. An impact of the severity of malnutrition on the cortical threshold was also observed. Prolongation of the cortical latency and central motor conduction time in malnourished children is indicative of motor conduction dysfunction. It has been established that after transcranial magnetic stimulation pyramidal tract cells of the motor cortex are excited either directly or trans-synaptically via tangentially oriented afferent fibres. ${ }^{10}{ }^{11}$ The conduction time of the muscle evoked potential from the cortex to the C7-C8 segments of the cervical spinal cord is about $5.0 \mathrm{~ms}$. Such short latencies can only be accomplished by the fastest conducting pyramidal tract cells- that is, giant Betz cells. ${ }^{12}$

It has been established that the conduction velocities in peripheral motor nerves reach adult values by 3 years of age. ${ }^{13}$ Histochemical studies of necropsy samples suggest that myelination of the corticospinal tract is complete by 3 years of age. ${ }^{14} \mathrm{~A}$ progressive increase in conduction velocity within the descending motor pathways has been reported until 11 years of age, when adult values are achieved. It has been suggested that an as yet uncharacterised process of maturation exists that leads to an apparent increase in the conduction velocity within the corticospinal tract beyond completion. This may be due to in part to the development and maturation of interneural synaptic connection, both at the cortex and the spinal cord. ${ }^{7}$ The clinical implication of this observation might explain the normal development of motor behaviour, which shows the continued acquisition of fine motor skills until the age of 8-10 years. ${ }^{15}{ }^{16}$ Our study showed significant dysfunction of conduction velocity in malnourished children.

Our preliminary observation of lowered cortical excitability and central motor dysfunction in malnourished children will be of great help in following up the functional assessment of these children with nutrition rehabilitation. We are currently assessing whether central motor conduction dysfunction may be reversed when malnutrition is treated. neur PM, Dastur DK. Pediatric malnutrition-a global EP, eds. Neurological sciences a , Sahani M, Bharucha problems. New Delhi: Interprint, 1989: 252-5.

2 Dobbing J, Hopewell JM, Lynch A. Vulnerability of developing brain VII. Permanent deficit of neurons in cerebral and cerebellar cortex following early mild undernutrition. Exp Neurol 1971;32;439-47.

3 Barker AT, Freeston IL, Jalinous R, Jarratt A. Magnetic Barker AT, Freeston IL, Jalinous R, Jarratt A. Magnetic
stimulation of human brain and peripheral nervous stimulation of human brain and peripheral nervous
system - an introduction and the results of an initial clinical evaluation. Neurosurgery 1987;20;100-9.

4 Ghai OP. Nutrition and nutritional disorder. Essential Ghai OP. Nutrition and nutritional disorder. Ese
pediatrics. 3rd Ed. New Delhi: Interprint, 1993: 51 .

5 Eisen A. Cortical and peripheral nerve magnetic stimulation methods. Clin Neurophysiol 1992;3:65-84.

6 Barker AT, Jalinous R, Freeston IL. Non-invasive magnetic stimulation of the human motor cortex. Lancet 1985;i: 1106-7.

7 Koh THHG, Eyre JA. Maturation of corticospinal tracts assessed by electromagnetic stimulation of the motor cortex. Arch Dis Child 1988;63:1347-52.

8 Hess CW, Milk KR, Murray NMF. Magnetic stimulation of human brain, facilitation of motor responses by voluntary contraction of ipsilateral and contralateral muscles with 71:235-40.

9 Flament D, Hall EJ, Lemon RN. The development of corticomotoneuronal projections investigated using magnetic brain stimulation in the infant macaque. f Physiol (Lond) brain stimulation

10 Day BL, Rothwell JC, Thompson PD, et al. Motor cortex stimulation in intact man II. Multiple descending volleys. Brain 1987;110:1191-289.

11 Edgley SA, Eyre JA, Lemon RN, Miller S. Excitation of corticospinal tract by electromagnetic and electrical stimulation of scalp in the macque monkey. F Physiol (Lond) 1990; 425:301-20

12 Mills KR, Murray NMF. Corticospinal tract conduction time in multiple sclerosis. Ann Neurol 1985;18:601-5. 13 Thomas JI, Lambert $\mathrm{EH}$. Ulnar nerve conduction velocity
and $\mathrm{H}$ reflex in infants and children. $\mathcal{f}$ Appl Physiol 1960;15:1-9.

14 Yakovier Pl, Lecours AR. The myelognetic cycles of regional maturation of brain. In: Miakowski A, ed. Regional development of the brain in early life. Oxford: Blackwell Scientific, 1967:3-70.

15 Illingworth RS. The development of the infant and young child, normal and abnormal. Edinburgh: Churchill Livingstone,
1966 .

16 Denckla MB. Development of motor co-ordination in normal children. Dev Med Child Neurol 1974;10:729-41. 